\title{
Topology Optimized Architectures with Programmable Poisson's Ratio over Large Deformations
}

\author{
Clausen, Anders; Wang, Fengwen; Jensen, Jakob Søndergaard; Sigmund, Ole; Lewis, Jennifer A.
}

Published in:

Advanced Materials

Link to article, DOI:

10.1002/adma.201502485

Publication date:

2015

Document Version

Peer reviewed version

Link back to DTU Orbit

Citation (APA):

Clausen, A., Wang, F., Jensen, J. S., Sigmund, O., \& Lewis, J. A. (2015). Topology Optimized Architectures with Programmable Poisson's Ratio over Large Deformations. Advanced Materials, 27(37), 5523-5527.

https://doi.org/10.1002/adma.201502485

\section{General rights}

Copyright and moral rights for the publications made accessible in the public portal are retained by the authors and/or other copyright owners and it is a condition of accessing publications that users recognise and abide by the legal requirements associated with these rights.

- Users may download and print one copy of any publication from the public portal for the purpose of private study or research.

- You may not further distribute the material or use it for any profit-making activity or commercial gain

- You may freely distribute the URL identifying the publication in the public portal 


\title{
WILEY-VCH
}

\section{Topology Optimized Architectures with Programmable Poisson's Ratio over Large Deformations}

Anders Clausen, Fengwen Wang, Jakob S. Jensen, Ole Sigmund* and Jennifer A. Lewis*

\author{
A. Clausen, Dr. F. Wang, Prof. O. Sigmund \\ Department of Mechanical Engineering \\ Solid Mechanics \\ Technical University of Denmark \\ 2800 Kgs. Lyngby, Denmark \\ E-mail: sigmund@mek.dtu.dk
}

Prof. J. S. Jensen

Department of Electrical Engineering

Centre for Acoustic-Mechanical Micro Systems

Technical University of Denmark

2800 Kgs. Lyngby, Denmark

Prof. J. A. Lewis

School of Engineering and Applied Sciences

Wyss Institute for Biologically Inspired Engineering

Harvard University Cambridge

MA 02138, USA

E-mail: jalewis@seas.harvard.edu

Keywords: material design, Poisson's ratio, topology optimization, 3D printing, large deformation

Structural materials are used in myriad applications, including aerospace, automotive, biomedical, and acoustics. Most materials have positive or zero Poisson's ratio, with cork serving as a well-known example of the latter type of behavior. The Poisson's ratio describes the relative amount a given material contracts transversally when stretched axially. Recently, artificial materials that exhibit a negative Poisson's ratio have been introduced. ${ }^{[1-3]}$ These auxetic materials expand transversally when axially stretched, seemingly defying the fundamental laws of nature. ${ }^{[1-3]}$ They exhibit enhanced mechanical properties, such as shear resistance, ${ }^{[4,5]}$ indentation resistance ${ }^{[6-9]}$ and extraordinary damping properties, ${ }^{[10]}$ making them well suited for targeted applications. To date, several types of auxetic materials have been introduced ${ }^{[2,3,11-20]}$. However, current embodiments suffer from two primary limitations: 


\section{WILEY-VCH}

(1) they only exhibit the desired response over a narrow range of strains (less than a few \%) and (2) they are difficult to manufacture in a scalable manner. ${ }^{[17,21-25]}$ While recent structures (e.g. chiral honeycombs, ${ }^{[14]}$ tilting square structures ${ }^{[24]}$ or Bucklicrystals ${ }^{[19]}$ ), for specific values of Poisson's ratio, exhibit near constant values over large strains, they are either not generalizable to other Poisson's ratio values or they exhibit low effective stiffness and/or must be pre-stressed to yield the desired performance.

Here, we combine topology optimization to programmably design their architecture with 3D printing to digitally fabricate the designs and validate against the numerically predicted behavior. Specifically, we create a new class of architected materials with programmable Poisson's ratios between -0.8 and 0.8 that display a nearly constant Poisson's ratio over large deformations of up to $20 \%$ or more. Figure 1 shows two representative examples of microstructures designed using topology optimization. ${ }^{[26]}$ The linear model that is applied by existing design methods (Figure 1a) assumes small deformations. By contrast, an emerging approach (Figure1b), described in detail in a recent study ${ }^{[26]}$ by Sigmund and coworkers, uses a geometrically nonlinear model and includes a requirement of a constant prescribed Poisson's ratio when straining the material. While both examples are designed to have a Poisson's ratio of -0.8 , the performance of the linearly designed material rapidly deteriorates when the material is strained more than a few percent (Figure 1c).

Mathematically, the optimization goal is defined as minimizing the error between the actual and the pre-defined value of Poisson's ratio over a range of discrete, nominal strain values up to $20 \% .{ }^{[26]}$ To ensure scalable fabrication of these architectures, several geometric constraints are imposed on the topology optimization design problem. A requirement of uniform structural features is implemented as a combination of imposing a minimum ${ }^{[27,28]}$ and a maximum length scale. The topology optimization step leads to a beam-like layout, which may be converted into a simplified design composed of a set of parameterized superellipses. 


\section{WILEY-VCH}

Uniform feature size is guaranteed by specifying a constant width for all the superellipses, while the length of each superellipse is controlled by design points (Figure S1). Using shape optimization to fine-tune the superellipse designs, we obtain architectures with uniform features having the desired response.

To demonstrate our approach, nine reference architectures are designed with equally dispersed and strain-independent constant Poisson's ratio values between -0.8 and 0.8 . Based on this discrete set of architectures, we derive a simple generic parameterization that provides a design guideline for any targeted value of the Poisson's ratio within the range of -0.8 to 0.8 . The details of the topology and shape optimization methodology as well as the derivation of the generic parameterization are provided in Supplementary Information and illustrated in Figure S2. We use direct ink writing (DIW) ${ }^{[29-31]}$, an extrusion-based 3D printing method, to fabricate these topologically optimized materials (Figure 2a). Inspired by initial results from the topology optimization process, we realized that materials with a negative or zero Poisson's ratio could be designed to ensure a continuous print path (Figure 2b), which is not generally obtained in topology optimized architectures. This ultimately ensures their scalable fabrication via multinozzle arrays ${ }^{[32]}$. The uniform feature size obtained with the superellipse approach allows printing with a constant nozzle speed and volumetric flow, thereby minimizing patterning errors. The structures with a strictly positive Poisson's ratio have a different topology requiring a modified printing strategy. The unit cell consists of round features connected by straight members (Figure 2c) and may be fabricated with a continuous print path by printing the connecting features with two passages of the nozzle, as indicated by the arrow in Figure 2c. This approach translates into a requirement of piecewise uniform feature size, with the double-printed features having correspondingly larger width, which is easily fulfilled using the superellipse approach.

We fabricated a complete series of topology optimized architectures that exhibit nearly constant values of Poisson's ratio over large deformations across nine equally dispersed 


\section{WILEY-VCH}

values ranging between -0.8 and 0.8 (Figure 3a-c). These samples (10-layers) are printed using a silicone-based elastomeric ink and then cured. Each sample is then subjected to uniaxial tensile testing (Figure 3e) and its performance is compared to the numerical prediction (Figure 3f). The deformation patterns for samples of the two extremal Poisson's ratio values (-0.8 and 0.8$)$ clearly demonstrate the large deformations associated with the expanding versus contracting behavior (Figure 3d and 3g). The definition of Poisson’s ratio used for design and validation is the negative ratio of the transverse strain, $\varepsilon_{2}$, to the longitudinal strain, $\varepsilon_{l}$ :

$v=\frac{-\varepsilon_{2}}{\varepsilon_{1}}$

The applied strain measure is the engineering strain, i.e., $\varepsilon=\Delta L / L_{0}$, where $\Delta L$ is the change in distance between two points initially separated by the distance $L_{0}$. The experimentally measured values are an average over the four (two by two) central unit cells. Despite minor deviations, the experimental results are in good agreement with the predicted behavior for both the negative and positive Poisson’s ratio designs. In the latter case, the experimental values are slightly below the numerical curve for large strains in all samples. This deviation likely arises to slight geometrical differences between the fabricated samples and the programmed designs, rather than flaws in the numerical model. For example, the initial (lower) layers deform slightly due to gravitational and viscous forces as subsequent (upper) layers are printed. The upper layers are drawn slightly inwards at sections with strong curvature due to viscous forces in the ink. Hence, the desired uniform feature size is not perfectly realized in those samples.

As noted earlier, the four optimized microstructures with positive Poisson's ratio share a very similar generic configuration (Figure 4a), while the five optimized microstructures with negative or zero Poisson’s ratio share another (albeit related) generic configuration (Figure 4b). To clearly demonstrate their configuration evolution versus Poisson’s ratio only 


\section{WILEY-VCH}

the skeletons of the optimized materials are shown. Based on the optimized design points for the nine reference designs, the microstructural configuration for any given Poisson's ratio within the interval $[-0.8,0.8]$ can be obtained by using a B-spline interpolation of the design points. The validity of this interpolation is illustrated in Figure 4c, where the total absolute performance error, summed up over the entire strain range, is displayed not only for the nine reference structures, but also for seven interpolated structures between each design point in both the positive and the negative range of Poisson's ratio. Figure 4c clearly shows that all designs perform well in a predictable fashion with only small variations.

In summary, by combining topology optimization with additive manufacturing, we have created a new class of architected materials. We have developed a simple geometric parameterization for the layout of the microstructural designs based on numerical optimization studies. We have fabricated materials architectures with programmable Poisson's ratio values ranging from -0.8 to 0.8 over deformations that are an order of magnitude greater than those observed previously. Our approach opens new avenues to the design and rapid fabrication of programmable materials possessing exotic properties.

\section{Experimental Section}

Silicone Ink: The ink is a polydimethylsiloxane (PDMS) silicone material, SE 1700 (Dow Corning), which consists of a 10:1:1 mix ratio of SE 1700 Clear Base, SE 1700 Catalyst (both Dow Corning) and silicone oil (viscosity 350 cSt ( $\left.25^{\circ} \mathrm{C}\right)$, Sigma-Aldrich Chemistry), respectively. The parts are mixed in a $15 \mathrm{~mL}$ container for $5 \mathrm{~min}$ at $2000 \mathrm{rpm}$ using a planetary mixer (Dual Asymmetric Centrifugal SpeedMixer, FlackTek Inc., DAC 600.2 VAC-P). For each test specimen, $6 \mathrm{~g}$ of mixed material is prepared.

3D Printing: The ink is loaded into a 10cc, luer-lock syringe (Nordson EFD Optimum) and centrifuged for $10 \mathrm{~min}$ at $3000 \mathrm{rpm}$ to remove air bubbles (Thermo Electron Corporation IEC Centra CL2 Centrifuge). The loaded syringe is placed on an Aerotech 3-axis positioning stage 


\section{WILEY-VCH}

(Aerotech, Inc.). Ink deposition is controlled pneumatically using an Ultimus V pressure box (Nordson EFD). The ink is printed through a $200 \mu \mathrm{m}$ luer-lock syringe tip (Nordson EFD) onto a glass plate covered with PTFE-coated aluminum foil to prevent adhesion (Bytac, SaintGobain). Print paths are generated from optimized designs by converting node positions into parameterized G-code scripts. Test specimens (10 layers with $136 \mu$ m layer thickness) are printed on a custom-designed 3-axis motion-controlled stage (Aerotech, Inc). Printed parts are cured at $100{ }^{\circ} \mathrm{C}$ for $4 \mathrm{~h}$.

Uniaxial Tensile Tests: For each of the fabricated architectures, a test specimen consisting of 12 (longitudinal) by 8 (transversal) unit cells ( $5 \mathrm{~mm}$ x $5 \mathrm{~mm}$ ) was printed. At both ends, a 15 $\mathrm{mm}$ grid is printed to allow mounting the sample for mechanical testing. For the tensile tests, the attachment grid at both ends is friction fastened between two pieces of purpose cut $6 \mathrm{~mm}$ acrylic plate, the latter held together using bolts and nuts. Each sample is tested in a vertical setup. The top end is attached to the z-stage of the Aerotech positioning system using screws. The bottom end is attached between two steel blocks held in place by gravity. The initial sample length (distance between acrylic plates of opposite ends) is measured using a digital caliper. The sample is strained at $2 \%$ increments between 0 and $20 \%$. Each step is monitored using a digital SLR camera (22.3 M Pixels Canon 5D Mark III with a Canon US Macro 100 mm objective) synchronized with the setup.

Image Analysis: Each photo from the strain test is converted to a contour plot using the MATLAB Image Processing Toolbox. Distances, measured in pixels between corresponding features, are tracked between images for all nominal strain values for each sample. Numerical simulations of the entire tensile test specimen revealed that boundary effects, such as constraints on both ends of the specimen, only cause minor deviations (less than 5\%) when evaluating the Poisson's ratio using the four unit cells located in the center of the specimen. Hence, experimental strain values used for computing the Poisson's ratio were determined as 


\section{WILEY-VCH}

an average over the four unit cells within the $2 \times 2$ array located in the center of each

specimen.

\section{Acknowledgements}

AC and FW contributed equally to this work. AC and OS acknowledge financial support from the Villum Foundation (the NextTop project) and DTU Mechanical Engineering. FW and JSJ acknowledge financial support from the ERC starting Grant through INNODYN. JAL acknowledges support from Army Research Office Award No. W911NF-13-0489. The experimental work was carried out in relation to AC's research stay at the Lewis Research Group during the Fall, 2014. We would like to acknowledge Joseph Muth and Dr. Jordan R. Raney from the Lewis group for valuable input on the experimental work and Dr. Lori Sanders for assistance on the photographic documentation.

\section{References}

[1] L. J. Gibson, M. F. Ashby, G. S. Schajer, C. I. Robertson, Proc. R. Soc. 1982, 382, 25.

[2] R. Lakes, Science 1987, 235, 1038.

[3] K. E. Evans, Endeavour 1991, 15, 170.

[4] J. Choi, R. Lakes, J. Mater. Sci. 1992, 27, 5375.

[5] J. Choi, R. Lakes, J. Mater. Sci. 1992, 27, 4678.

[6] R. Lakes, K. Elms, J. Compos. Mater. 1993, 27, 1193.

[7] N. Chan, K. E. Evans, J. Cell. Plast. 1998, 34, 231.

[8] K. L. Alderson, A. P. Pickles, P. J. Neale, K. E. Evans, Acta Metall. Mater. 1994, 42, 2261.

[9] K. L. Alderson, A. Fitzgerald, K. E. Evans, J. Mater. Sci. 2000, 35, 4039.

[10] C. Chen, R. Lakes, J. Eng. Mater. Technol. 1996, 118, 285.

[11] E. Friis, R. Lakes, J. Park, J. Mater. Sci. 1988, 23, 4406.

[12] K. L. Alderson, K. E. Evans, Polymer 1992, 33, 4435.

[13] G. W. Milton, J. Mech. Phys. Solids 1992, 40, 1105.

[14] D. Prall, R. Lakes, Int. J. Mech. Sci. 1997, 39, 305.

[15] R. Lakes, R. Witt, Int. J. Mech. Eng. Educ. 2002, 30, 50.

[16] T. Bückmann, N. Stenger, M. Kadic, J. Kaschke, A. Frölich, T. Kennerknecht, C. Eberl, M. Thiel, M. Wegener, Adv. Mater. 2012, 24, 2710. 


\section{WILEY-VCH}

[17] U. D. Larsen, O. Sigmund, S. Bouwstra, J. Microelectromech. Syst. 1997, 6, 99.

[18] K. Bertoldi, P. M. Reis, S. Willshaw, T. Mullin, Adv. Mater. 2010, 22, 361.

[19] S. Babaee, J. Shim, J. C. Weaver, E. R. Chen, N. Patel, K. Bertoldi, Adv. Mater. 2013, 25, 5044.

[20] T. Bückmann, R. Schittny, M. Thiel, M. Kadic, G. W. Milton, M. Wegener, New J. Phys. 2014, 16, 033032.

[21] O. Sigmund, Int. J. Solids Struct. 1994, 31, 2313.

[22] E. Andreassen, J. S. Jensen, Struct. Multidiscip. Optim. 2013, 49, 695.

[23] O. Sigmund, S. Torquato, I. A. Aksay, J. Mater. Res. 2011, 13, 1038.

[24] O. Sigmund, Mech. Mater. 1995, 20, 351.

[25] E. Andreassen, B. S. Lazarov, O. Sigmund, Mech. Mater. 2014, 69, 1.

[26] F. Wang, O. Sigmund, J. S. Jensen, J. Mech. Phys. Solids 2014, 69, 156.

[27] F. Wang, B. S. Lazarov, O. Sigmund, Struct. Multidiscip. Optim. 2011, 43, 767.

[28] M. Zhou, B. S. Lazarov, F. Wang, O. Sigmund, Comput. Methods Appl. Mech. Eng. 2015, 293, 266.

[29] J. E. Smay, J. Cesarano III, J. A. Lewis, Langmuir 2002, 18, 5429.

[30] G. M. Gratson , M. Xu, J. A. Lewis, Nature 2004, 428, 386.

[31] B. Y. Ahn, E. B. Duoss, M. J. Motala, X. Guo, S.-I. Park, Y. Xiong, J. Yoon, R. G. Nuzzo, J. A. Rogers, J. A. Lewis, Science 2009, 323, 1590.

[32] C. J. Hansen, R. Saksena, D. B. Kolesky, J. J. Vericella, S. J. Kranz, G. P. Muldowney, K. T. Christensen, J. A. Lewis, Adv. Mater. 2013, 25, 96. 

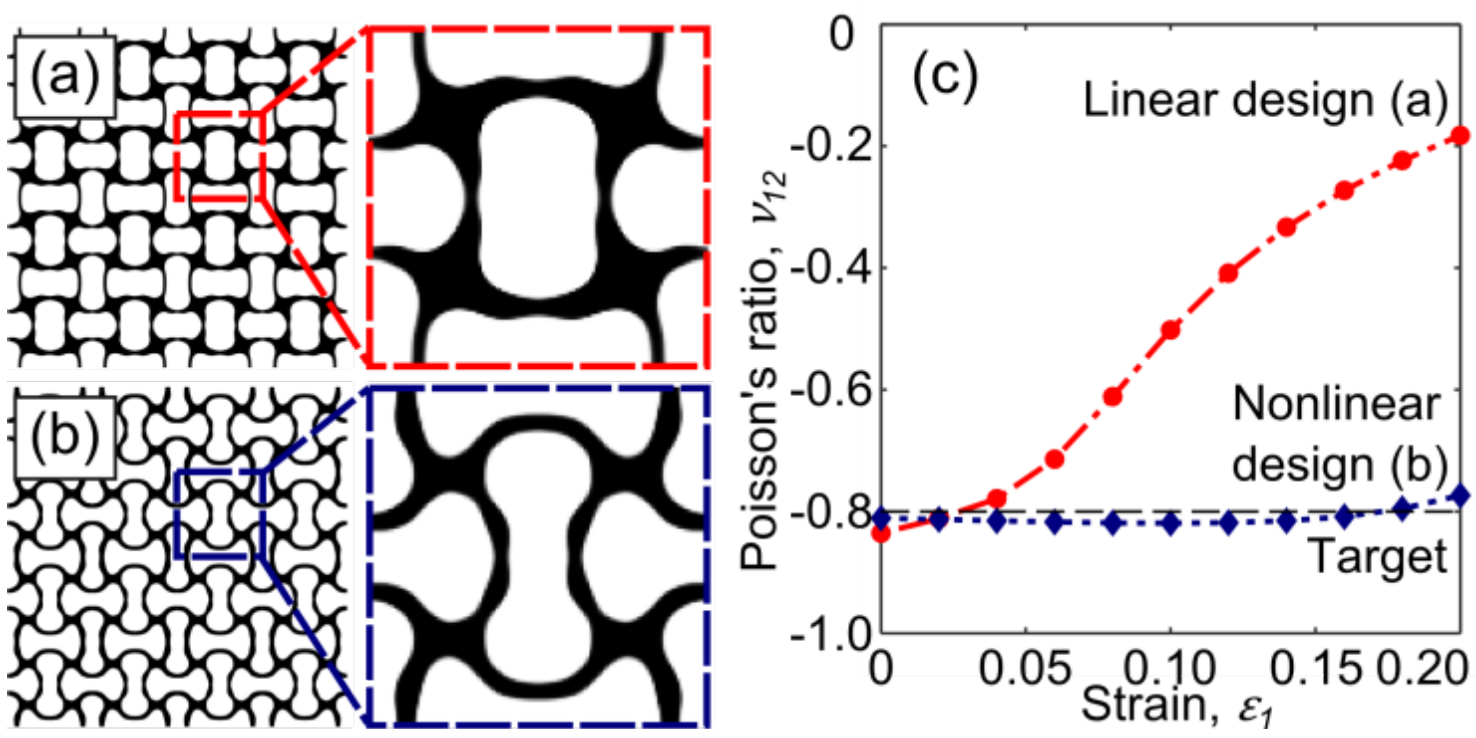

Figure 1. Topology optimized microstructures for auxetic materials via (a) linear and (b) geometrical nonlinear modeling. (c) Strain-dependent behavior of Poisson's ratio for large deformations for these respective materials.
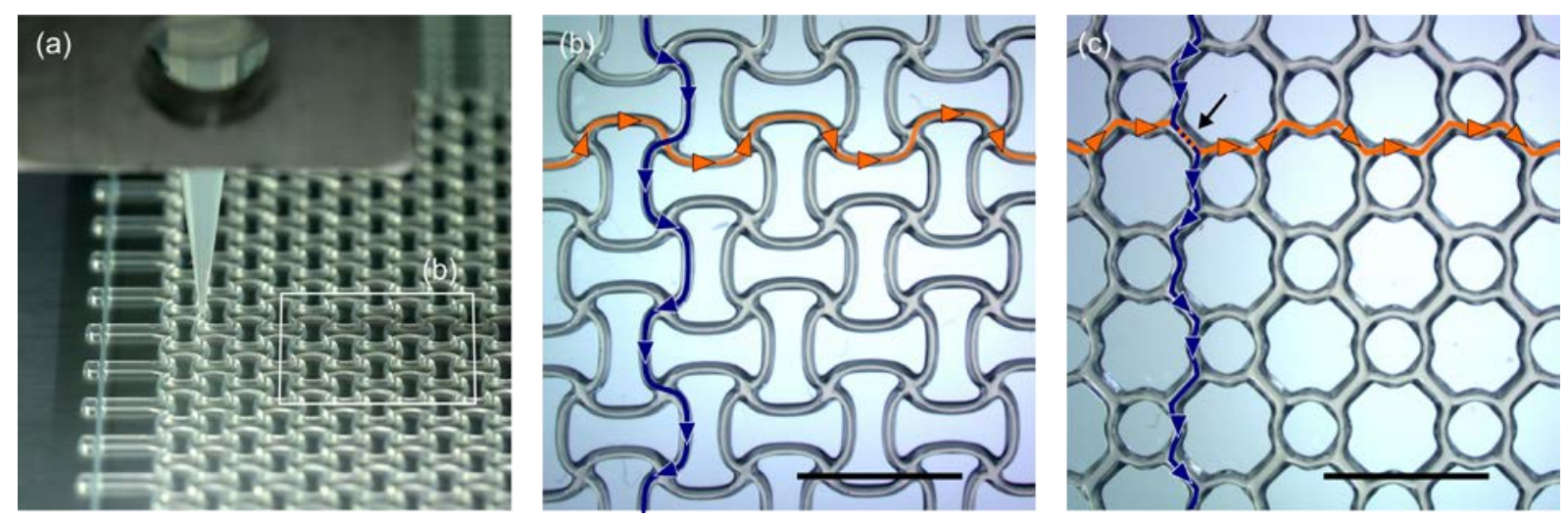

Figure 2. 3D printing method and constraints. (a) Optical image of the fabrication of PDMSbased architectures using direct ink writing. (b) Print path (indicated by superposed solid lines with triangle markers) for these structures for negative and zero Poisson's ratio. (c) Print path for positive Poisson's ratio. For segments with overlapping print paths (indicated by arrow), the features are correspondingly wider. Scale bars in (b) and (c) are $5 \mathrm{~mm}$. 

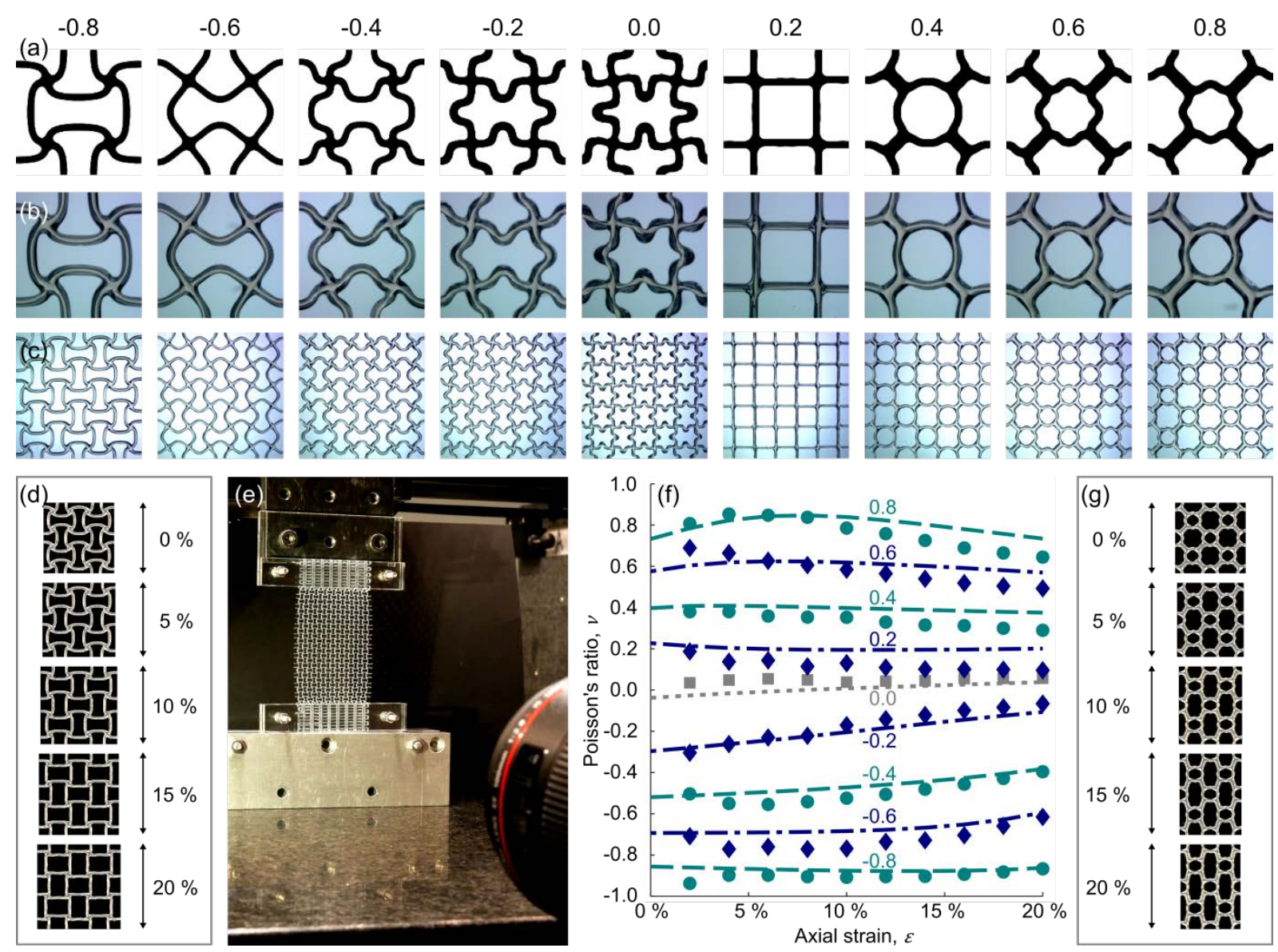

Figure 3. Topology and shape optimized architectures. (a) Designed and (b) printed unit cells for a programmable range of Poisson's ratio values. (c) 3x3 unit cells of the corresponding PDMS-based architectures. (d) Deformation pattern of the $2 x 2$ central unit cells corresponding to given longitudinal nominal strain values for $v=-0.8$ (in (g) for $v=0.8$ ). (e) Experimental setup for tensile tests. (f) Comparison between experimental (points) and numerically predicted (dashed lines) results. Unit cell size for all architectures in (a-g) is 5 $\mathrm{mm}$.
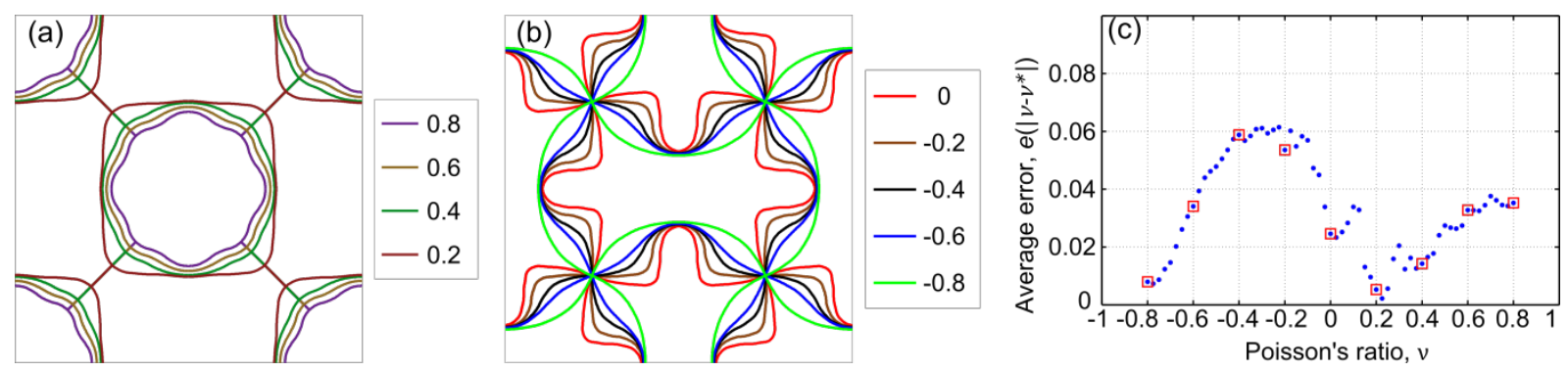

Figure 4. Generic parameterization of engineered architectures. Visual comparison of the unit cell layout for (a) positive and (b) negative or zero Poisson's ratio. (c) Average absolute error for parameterized designs for any given Poisson's ratio. The red square points indicate the nine optimized reference structures. 


\section{WILEY-VCH}

\section{Supplementary Information}

\section{Topology Optimized Architectures with Programmable Poisson’s Ratio over Large Deformations}

Anders Clausen, Fengwen Wang, Jakob S. Jensen, Ole Sigmund* and Jennifer A. Lewis*

\section{Topology optimization}

Topology optimization can efficiently be employed to design structural materials with a programmable Poisson's ratio values. A design domain defining the unit cell is discretized using a number of elements, defining both the design mesh and the finite element mesh used to model the periodic microstructure. The material occupation in each element, $e$, is controlled by a design variable, $\boldsymbol{\rho}_{\boldsymbol{e}}$, which can take values between 0 and 1 . By iteratively changing the design variables based on gradient information, the microstructural layout can be optimized to achieve the prescribed Poisson’s ratio. Exact topology optimization formulation and procedures are described in detail in a recent study. ${ }^{[25]}$

To ensure scalable fabrication of the architectures, we first imposed geometric constraints like minimum ${ }^{[26,27]}$ and a maximum ${ }^{[28]}$ length scale on the topology optimization problem, leading to beam-like layouts with only minor variations in feature size. Inspired by these architectures, we formulated a shape optimization step by parameterizing the previously optimized microstructures using a set of superellipses to ensure completely uniform member sizes. In this second optimization step, any finite element, $e$, is defined as solid $\left(\rho_{e}=1\right)$ or void $\left(\rho_{e}=0\right)$, depending on whether its center $\left(x_{e}, y_{e}\right)$ is located within one of the superellipses or not. Thereby, the material occupation of a given element, $e$, can be described by

$\rho_{e}=\max \left(S_{i}\left(x_{e}, y_{e}\right)\right), i=1 . . N$ 


\section{WILEY-VCH}

where $S_{i}$ indicates whether element $e$ is inside the $i^{\text {th }}$ superellipse, controlled by the $i^{\text {th }}$ and $(i+1)^{\text {th }}$ design points as illustrated in Supplementary Fig. 1a. $S_{i}\left(x_{e}, y_{e}\right)=1$ indicates that element $e$ is within the $i^{\text {th }}$ superellipse, and $S_{i}\left(x_{e}, y_{e}\right)=0$ indicates that the element is outside. Mathematically, $S_{i}$ is given as

$S_{i}\left(x_{e}, y_{e}\right)=1-\frac{1}{1+e^{\left(-\beta\left(\left(\frac{A}{a^{i}}\right)^{100}+\left(\frac{B}{b^{i}}\right)^{100}-1\right)\right)}}$,

where $\beta$ is chosen as 50 , and the parameters $A$ and $B$ are calculated by

$A=\left(x_{e}-\left(x^{i}+x^{i+1}\right) / 2\right) \cos \alpha+\left(y_{e}-\left(y^{i}+y^{i+1}\right) / 2\right) \sin \alpha$

and

$B=-\left(x_{e}-\left(x^{i}+x^{i+1}\right) / 2\right) \sin \alpha+\left(y_{e}-\left(y^{i}+y^{i+1}\right) / 2\right) \cos \alpha$

Here, $\alpha$ is the angle of the line connecting the $i^{\text {th }}$ and $(i+1)^{\text {th }}$ design points. It is calculated by $\alpha=\tan ^{-1}\left(\frac{y^{i+1}-y^{i}}{x^{i+1}-x^{i}}\right)$

The semi-diameter $a^{i}$ is fixed to be half of the desired feature size and the semidiameter $b^{i}$ is defined by half of the distance between the two design points. Based on this design parameterization, materials with pre-defined Poisson's ratio are fine-tuned by changing the locations of design points, using a gradient-based optimization algorithm.

The unit cell size of all optimized superellipse-based designs is $5.0 \mathrm{~mm}$. Designs with a negative or zero Poisson's ratio have constant feature size of $300 \mu \mathrm{m}$, or $a_{1}{ }^{i}=150 \mu \mathrm{m}$. For comparison, a design with positive Poisson's ratio $(v=0.4)$ is shown in Supplementary Fig. 1b. The connecting features located along diagonals are represented by superellipses with an increased semi-diameter $\left(a_{2}{ }^{i}=275 \mu \mathrm{m}\right)$ to reflect the two passages of the nozzle in the fabrication process as illustrated in Fig. 2c in the main article. The semi-diameter $a_{2}{ }^{i}$ was determined experimentally based on the print parameters giving the value $a_{1}{ }^{i}$. The reason that $a_{2}{ }^{i}$ is not exactly the double value of $a_{1}{ }^{i}$ (we found that $a_{2}{ }^{i}<2 a_{1}{ }^{i}$ ) is that at the second 


\section{WILEY-VCH}

passage of the nozzle, the PDMS is extruded into the already printed material from the first passage, giving rise to a small viscous resistance and thereby a lower extrusion rate.

\section{Generic parameterization of microstructural architectures}

Nine reference architectures with equally dispersed Poisson's ratio values between -0.8 and 0.8 are created using the combined topology and shape optimization approach. The microstructural configuration for a material with any prescribed Poisson's ratio within this range can be obtained by interpolating the design points from the reference architectures. Specifically, we use the Matlab cubic spline interpolation function "csapi” to obtain the interpolation functions.

The microstructural configuration for a material with any Poisson's ratio within the range $[-0.8,0]$ is interpolated using the five reference architectures with Poisson's ratio smaller than or equal to zero. Supplementary Fig. 2 illustrates the cubic spline interpolation of the $y$-coordinate of a single design point. Similarly, the microstructural architecture for a material with a Poisson's ratio within the range $[0.2,0.8]$ is interpolated using the four reference architectures with positive Poisson's ratio. Configurations for the intermediate range $[0,0.2]$ are interpolated using the two reference architectures with Poisson's ratios of 0 and 0.2 . 


\section{WILEY-VCH}

(a)

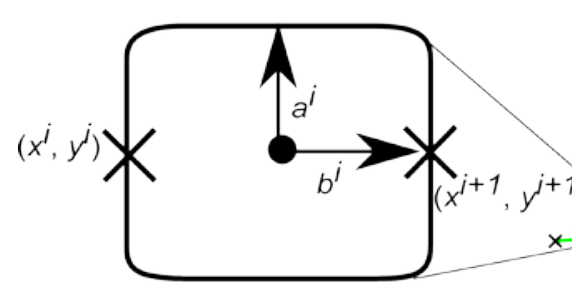

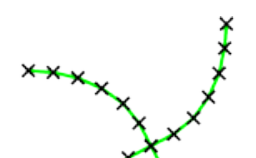

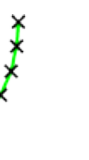

$\stackrel{x}{x}_{x}^{x}$

$x^{x} x^{x^{x x x}}$
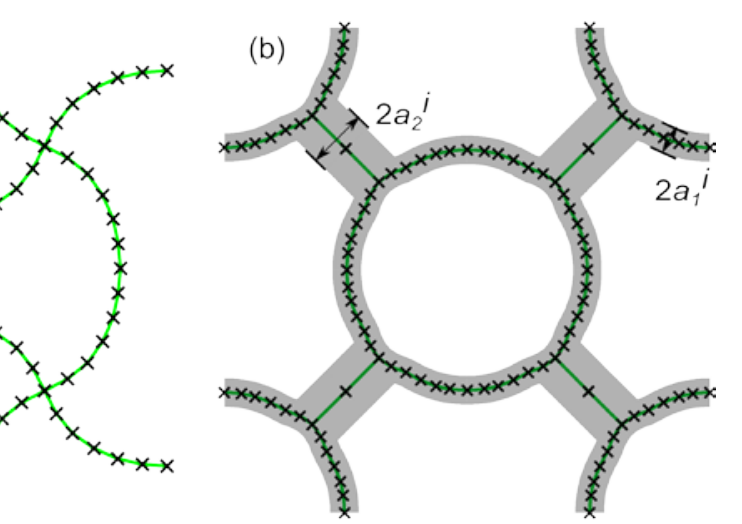

Figure S1. (a) Illustration of the design parameterization based on superellipses used in the shape optimization. (b) Illustration of the superellipse-based design parameterization for reference architecture with positive Poisson’s ratio $(v=0.4)$.

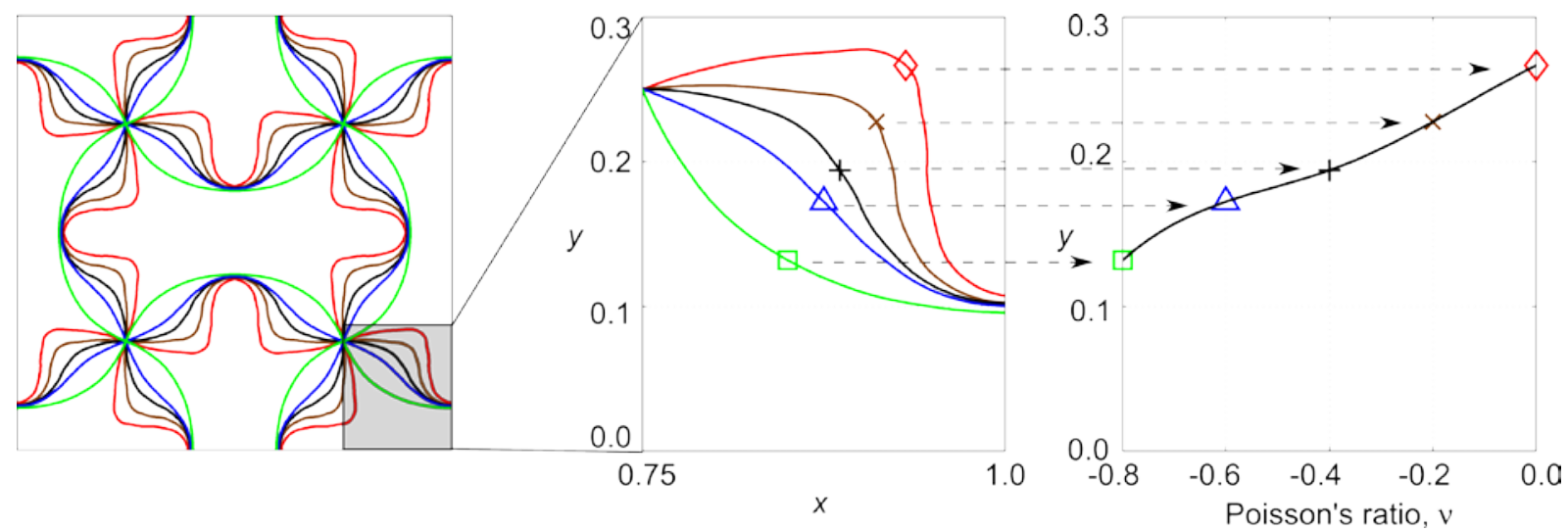

Figure S2. Representative example of the $y$-coordinate interpolation of a single design point as a function of Poisson's ratio. 